DOI: 10.1002/adma.201700767

Article type: Communication

\title{
Game Changer: A Multi-functional Perovskite Exhibiting Giant Ferroelectricity and Narrow Bandgap with Potential Application in a Truly Monolithic Multi-energy Harvester or Sensor
}

Yang Bai*, Pavel Tofel, Jaakko Palosaari, Heli Jantunen and Jari Juuti

Dr. Y. Bai, J. Palosaari, Prof. H. Jantunen, Dr. J. Juuti

Microelectronics Research Unit, Faculty of Information Technology and Electrical

Engineering, University of Oulu, FI-90014 Oulu, Finland

E-mail: yang.bai@oulu.fi

Dr. P. Tofel

CEITEC - Central European Institute of Technology, Technicka 3058/10, CZ-61600 Brno, Czech Republic

Keywords: multi-source energy harvesting, piezoelectric, pyroelectric, photovoltaic, multifunctional

Abstract: An $\mathrm{ABO}_{3}$-type perovskite solid-solution, $\left(\mathrm{K}_{0.5} \mathrm{Na}_{0.5}\right) \mathrm{NbO}_{3}$ (KNN) doped with 2 mol.\% $\mathrm{Ba}\left(\mathrm{Ni}_{0.5} \mathrm{Nb}_{0.5}\right) \mathrm{O}_{3-\delta}(\mathrm{BNNO})$ is reported in this communication. Such a composition yields a much narrower bandgap $(\sim 1.6 \mathrm{eV})$ compared to the parental composition - pure KNN - and other widely used piezoelectric and pyroelectric materials (e.g. $\left.\mathrm{Pb}(\mathrm{Zr}, \mathrm{Ti}) \mathrm{O}_{3}, \mathrm{BaTiO}_{3}\right)$. Meanwhile, it exhibits the same large piezoelectric coefficient as that of $\mathrm{KNN}\left(\sim 100 \mathrm{pC} \mathrm{N}^{-1}\right)$ and a much larger pyroelectric coefficient $\left(\sim 130 \mu \mathrm{C} \mathrm{m}^{-2} \mathrm{~K}^{-1}\right)$ compared to the previously reported narrow bandgap material $\left(\mathrm{KNbO}_{3}\right)_{1-\mathrm{x}}-\mathrm{BNNO}_{\mathrm{x}}$. The unique combination of these excellent ferroelectric and optical properties opens the door to the development of multi-source energy harvesting or multi-functional sensing devices for the simultaneous and efficient conversion of solar, thermal and kinetic energies into electricity simultaneously and efficiently in a single material. Individual and comprehensive characterizations of the optical, ferroelectric, piezoelectric, pyroelectric and photovoltaic properties are investigated with single and co-existing energy sources. No degrading interaction between ferroelectric and photovoltaic behaviors was observed. This composition may fundamentally change the working principles of state of the art hybrid energy harvesters and sensors, and thus significantly increase the unit 


\section{WILEY-VCH}

volume energy conversion efficiency and reliability of energy harvesters in ambient environments.

Various energy harvesting (EH) techniques have been investigated in recent decades in order to overcome the shortcomings of batteries in terms of lifespan, overall cost-effectiveness and chemical safety. ${ }^{[1]}$ However, the power level and stability provided by a single-source energy harvester are often insufficient for practical applications. In order to address this issue, various hybrid energy harvesters have been developed and investigated. ${ }^{[2-4]}$ However, as such hybrid energy harvesters have mostly been simple physical combinations of individual harvesters made from different materials/structures, the effective size of the entire system can become much larger than its individual counterparts. ${ }^{[2,3]}$ In such a case one has to compromise either on the number of simultaneously harvested energy sources or on the space taken by different energy harvesting components. ${ }^{[5]}$ This compromise usually leads to the loss of the advantage of energy harvesters over batteries. A similar situation may occur in hybrid sensors.

One method to solve the problem is to design or discover a single composition/material which enables the simultaneous harvesting/detection of multiple energy sources. At the same time the individual conversion efficiency of the material for each energy source should be neither reduced nor interrupted in this multi-task performance. This requires different energy conversion effects exhibited by the same material to be independent of each other, or coupled but working in the same direction, and to be functional simultaneously. This communication reports a perovskite solid-solution, $\left(\mathrm{K}_{0.5} \mathrm{Na}_{0.5}\right) \mathrm{NbO}_{3} \quad(\mathrm{KNN})$ doped by 2 mol.\% $\mathrm{Ba}\left(\mathrm{Ni}_{0.5} \mathrm{Nb}_{0.5}\right) \mathrm{O}_{3-\delta}(\mathrm{BNNO})$ sintered at $1165{ }^{\circ} \mathrm{C}$ - exclusively abbreviated as the $\mathrm{KNBNNO}$ throughout this paper. The material shows large ferroelectric, piezoelectric and pyroelectric properties together with a narrow bandgap. The piezoelectric, pyroelectric and photovoltaic responses are proved not to hinder each other to any recognizable extent. They are functional 


\section{WILEY-VCH}

without any observed reduction of the individual efficiencies for the same volume/area covered by the same pair of electrodes.

Figure 1(a) shows the XRD pattern (obtained at room temperature) of the sintered ceramic sample of the KNBNNO. The pattern of a pure KNN ceramic sample is also included as a reference. Certain peaks analyzed through the Rietveld refinement are marked for the phase determination. The ceramic formed a single orthorhombic perovskite $\left(\mathrm{ABO}_{3}\right.$-type $)$ phase, consistent with its parental composition $-\mathrm{KNN}$. The calculated lattice parameters are also labeled in Figure 1(a). Details of the phase transitions are given in Figure S1 in the supporting information, and crystal structure investigation may be obtained from the Fachinformationszentrum Karlsruhe, 76344 Eggenstein-Leopoldshafen (Germany), on quoting the depository number CSD-432352.

Figure 1(b) shows the optical absorption and reflection spectra obtained from the thick-film samples, where $R$ is reflectance, $h$ is Planck's constant and $v$ is the light frequency. Theoretically $F(R)=(1-R)^{2} \cdot(2 R)^{-1}$, which has been used as a reliable factor and simple method to determine bandgaps of solid materials. ${ }^{[6]}$ The samples exhibited a narrow bandgap of $1.6 \pm 0.5 \mathrm{eV}$, which is very close to the photon energy $(1.65 \mathrm{eV})$ of the visible light spectrum. ${ }^{[7]}$ This indicates that most visible and ultra-violet parts of the solar spectrum could be absorbed by the reported composition. As mentioned, only 2 mol.\% BNNO (equivalent to 0.17 mol.\% $\mathrm{Ni}^{2+}$-oxygen vacancy combinations) was doped into the $\mathrm{KNN}$, and the bandgap of the KNBNNO was dramatically decreased by nearly $2.5 \mathrm{eV}$ from $>4 \mathrm{eV}$ of its parental $\mathrm{KNN} .{ }^{[8]} \mathrm{In}$ comparison, in a previous report 10-50 mol.\% BNNO (equivalent to $0.83-4.14$ mol.\% $\mathrm{Ni}^{2+}-$ oxygen vacancy combinations) was doped into the parental composition $-\mathrm{KNbO}_{3}{ }^{[7]}$ With this large amount of doping, a similar level of bandgap (1.1-2.0 eV) was obtained from the doped composition, compared to approximately $4 \mathrm{eV}$ from the parental $\mathrm{KNbO}_{3} .{ }^{[7]}$ Ferroelectric perovskites have been proved to be true semiconductors rather than typical insulators. ${ }^{[9]}$ In these materials, the spontaneous polarizations act as internal $\mathrm{p}-\mathrm{n}$ junctions (defined as self- 


\section{WILEY-VCH}

junctions). ${ }^{[10]}$ These charge carriers largely determine the band bending at the surfaces and interfaces and thus the photovoltaic behavior of the ferroelectric perovskites. ${ }^{[10]}$ In addition, the bandgaps of ferroelectric perovskites can be significantly reduced by up to $1.2 \mathrm{eV}$ through phase transitions alone, where the phase with a larger unit cell distortion (anisotropy) tends to lead to wider bandgaps. ${ }^{[11]}$ The bandgaps and remanent polarizations $(\mathrm{Pr})$ of the KNN compositions doped with different amounts of BNNO are summarized in Table 1. As can be seen, the bandgap experiences a sharp decrease between the pure $\mathrm{KNN}$ and the KNBNNO, and then remains approximately at the same level until $10 \mathrm{~mol} . \%$ of BNNO is doped, when another recognizable decrease is observed. It is believed that the $\mathrm{Ni}^{2+}$-oxygen vacancy combinations $\left(\mathrm{Ni}^{2+}-\mathrm{V}_{\mathrm{O}}\right)$ elevate electronic states in the gap of the parental $\mathrm{KNbO}_{3}{ }^{[5,7]}$ Similarly, this also happens in $\mathrm{KNN}$, as proved in this paper. In the $\mathrm{KNBNNO}$, the $\mathrm{Ni}^{2+}-\mathrm{V}_{\mathrm{O}}$ helped to reduce the bandgap. Meanwhile, the relatively small amount of BNNO kept the unit cell distortion at a low level. These two mechanisms may result in a significantly reduced bandgap compared to that of the pure $\mathrm{KNN}$. With an increasing amount of $\mathrm{BNNO}$ doping, the $\mathrm{Ni}^{2+}-\mathrm{V}_{\mathrm{O}}$ may provide a higher concentration of charge carriers which in principle should further reduce the bandgap. However, the increasing level of unit cell distortion caused by large $\mathrm{Ba}$ atoms is likely to lead to a widened bandgap, which counteracts the bandgap reduction resulting from the larger $\mathrm{Ni}^{2+}-\mathrm{V}_{\mathrm{O}}$ concentrations. Therefore, a minor amount of $\mathrm{Ni}^{2+}-\mathrm{V}_{\mathrm{O}}(0.17 \mathrm{~mol} . \%)$ may be enough to significantly elevate electronic states in the gap of the parental KNN, compared to the $0.83-4.17$ mol.\% used in a $\left(\mathrm{KNbO}_{3}\right)_{1-\mathrm{x}-\mathrm{BNNO}_{\mathrm{x}} \text { composition. }}{ }^{[7]}$ The reduced concentration of oxygen vacancies in the KNBNNO tends to cause a smaller domain wall pinning effect. ${ }^{[12]}$ This was preferred in order to retain the giant ferroelectric, piezoelectric and pyroelectric properties of the parental composition as much as possible because a larger oxygen vacancy concentration degrades these properties. ${ }^{[5]}$ It can then help to better balance them with the photovoltaic property. ${ }^{[13]}$ 


\section{WILEY-VCH}

Figure 1(c) shows the ferroelectric hysteresis loops (FE loops) measured at $0.1 \mathrm{~Hz}, 1 \mathrm{~Hz}$ and $10 \mathrm{~Hz}$ and the converse effect piezoelectric coefficient $\left(d_{33}\right)$ with different electric fields of the unpoled ceramic samples of the KNBNNO. Details of the parameters applied in the measurements are given in Table $\mathbf{S 1}$ in the supporting information. Both the polarization and $d_{33}$ values saturated when the maximum electric field of $\pm 90 \mathrm{kV} \mathrm{cm}^{-1}$ was applied. The maximum polarization $\left(26 \pm 1 \mu \mathrm{C} \mathrm{cm}^{-2}\right)$ reached the same level as that of the pure $\mathrm{KNN}$ at the same temperature and with the same electric field. ${ }^{[14]}$ Compared to the hysteresis loop measured at $1 \mathrm{~Hz}$, slower $(0.1 \mathrm{~Hz})$ and faster $(10 \mathrm{~Hz})$ signals induced about $2.3 \%$ increase and decrease in the maximum polarization, respectively, reflecting the pinning effect caused by oxygen vacancies. ${ }^{[5]}$ Meanwhile, in the case of the $P_{r}$ of about $11 \mu \mathrm{C} \mathrm{cm}{ }^{-2}$, half of the value of the pure KNN was obtained. ${ }^{[14]}$ This may be because of the domain wall pinning effect and the reduced asymmetry of the unit cells compared to that of the $\mathrm{KNN}$ (see the lattice parameters shown in Figure 1(a)). However, as the concentration of oxygen vacancies was not significantly large and the phase remained as orthorhombic, only $50 \%$ of the $P_{r}$ was sacrificed in the KNBNNO samples compared to the KNN. In contrast, the $P_{r}$ of the previously reported $\left(\mathrm{KNbO}_{3}\right)_{0.9^{-}}$ $\mathrm{BNNO}_{0.1}$ was nearly $80 \%$ smaller than that of the un-doped $\mathrm{KNbO}_{3}$ (at room temperature) due to the 4 times higher calculated concentration of oxygen vacancies $(0.83 \mathrm{~mol} \%$ for the $\left(\mathrm{KNbO}_{3}\right)_{0.9}-\mathrm{BNNO}_{0.1}$ compared to $0.17 \mathrm{~mol} . \%$ for the KNBNNO). ${ }^{[5,7,15]}$ An FE loop measured from a thick-film sample of the KNBNNO at $0.1 \mathrm{~Hz}$ is also shown in Figure 1(c). Thick-films were fabricated by directly reducing the thickness of the ceramic bulk samples that had FE loops with maxima and remanent polarizations very similar to their thicker counterparts. Therefore, the overall properties of the thick-films were estimated to be little different from those of the thicker bulk counterpart. The $d_{33}$ value with a quasi-zero electric field $\left(d_{33}+0\right.$, referred to $d_{33}$ at a small positive electric field) was $100 \pm 5 \mathrm{pm} \mathrm{V}^{-1}$ (i.e. $\mathrm{pC} \mathrm{N}^{-1}$ ). Although the $P_{r}$ was decreased compared to that of the pure KNN, the same $d_{33}$ was achieved in the 


\section{WILEY-VCH}

KNBNNO ceramics when the samples were sufficiently poled $\left(d_{33}=80-110 \mathrm{pC} \mathrm{N}^{-1}\right.$ for the pure KNN). ${ }^{[14]}$ This implies that the piezoelectric response was not substantially affected by the microstructural defects (oxygen vacancies or defect dipoles e.g. $\mathrm{Ni}^{2+}-\mathrm{V}_{\mathrm{O}}$ ). On the contrary, the $d_{33}$ of the previously reported $\left(\mathrm{KNbO}_{3}\right)_{0.9}-\mathrm{BNNO}_{0.1}$ was only about $20 \mathrm{pm} \mathrm{V}^{-1}$ compared to 90 $110 \mathrm{pC} \mathrm{N}^{-1}$ for the pure $\mathrm{KNbO}_{3}$, where the piezoelectric effect was seriously restricted by nonnegligible oxygen vacancies. ${ }^{[5,15]}$ The $g_{33}$ and $d_{33} \cdot g_{33}$, two of the key parameters related to sensing and/or energy harvesting, were calculated as $11.9 \pm 0.8 \times 10^{-3} \mathrm{Vm} \mathrm{N}^{-1}$ and $1.2 \pm 0.2 \times 10^{-12} \mathrm{~m}^{2} \mathrm{~N}^{-1}$ from Equation 1 and 2, respectively $\left(\varepsilon_{r}\right.$ is permittivity of the sample, $\varepsilon_{0}$ is the vacuum permittivity). They were, respectively, 3 times and 13 times those of the previously reported $\left(\mathrm{KNbO}_{3}\right)_{0.9}-\mathrm{BNNO}_{0.1}{ }^{[5]}$ These very different properties of the $\left(\mathrm{KNbO}_{3}\right)_{0.9}$ $\mathrm{BNNO}_{0.1}$ and the $\mathrm{KNBNNO}$ were because the former composition had an excessive amount of BNNO which significantly deteriorated the ferroelectric properties due to the largely reduced asymmetry of the unit cell and the increased domain wall pinning effect. By contrast, in the latter composition the amount of $\mathrm{Ni}^{2+}-\mathrm{V}_{\mathrm{O}}$ was successfully limited to the smallest amount possible that was still enough to provide a narrow bandgap, whilst retaining the best possible ferroelectric response due to a relatively large asymmetry of the unit cell. The resistivities obtained together with the above measurements are summarized in Table 1. Given the narrow bandgap, the relatively large resistivity made the leakage current of the KNBNNO very weak (absolute value $<1 \mu \mathrm{A}$, current density $<20 \mathrm{~mA} \mathrm{~m}^{-2}$ ) and did not affect the effectiveness of poling.

$g_{33}=d_{33} \cdot \varepsilon_{r}{ }^{-1} \cdot \varepsilon_{0}{ }^{-1}$

$d_{33} \cdot g_{33}=d_{33}^{2} \cdot \varepsilon_{r}^{-1} \cdot \varepsilon_{0}^{-1}$

Figure 1(d) shows the pyroelectric current density $\left(J_{\text {pyro }}\right)$ generated from a temperature fluctuation in the range of $42-48^{\circ} \mathrm{C}$ measured with the poled ceramic samples of the KNBNNO. The reversed connection made the values and polarities of the pyro-currents completely 


\section{WILEY-VCH}

inverted compared to those of the forward connection, indicating a real pyroelectric response. The pyroelectric coefficient $(\gamma)$ was calculated using Equation 3 , where $i_{p}$ is the pyro-current $\left(i_{p}\right.$ $\left.=J_{p} \cdot S\right), S$ is the surface area of the sample covered by both electrodes, $T$ is the temperature, and $t$ is the time. ${ }^{[10]}$ The calculated $\gamma$ of the KNBNNO was $128 \pm 6 \mu \mathrm{C} \mathrm{m}^{-2} \mathrm{~K}^{-1}$. This value is very close to that of the pure $\mathrm{KNN}\left(140 \mu \mathrm{C} \mathrm{m}^{-2} \mathrm{~K}^{-1}\right) \cdot{ }^{[14]}$ It may imply that once the samples were well poled (assuming most switchable domains were aligned), the oxygen vacancies had only minor influence on the domain wall mobility with temperature (pyroelectric effect). The energy conversion efficiency of the KNBNNO via the pyroelectric effect is estimated to be very similar to that of pure KNN based on Equation 3.

$\gamma=i_{p} \cdot S^{-1} \cdot\left(\frac{d T}{d t}\right)^{-1}$

Table 1 summarizes the material and functional properties of KNN doped with different amounts of BNNO and sintered at different temperatures. With increased BNNO (oxygen vacancy concentrations), the $P_{r}$ was dramatically decreased, leading to the same rapid decrease in $d_{33}$ and $\gamma$. This was expected according to the above discussions. For the 2 mol.\% of BNNO doped composition, the higher sintering temperature tended to reduce the resistivity by a large extent. This occurred as a consequence of potassium volatilization above $800{ }^{\circ} \mathrm{C}$ which increases at higher temperatures. ${ }^{[16]}$ The $\mathrm{K}^{+}$vacancies increased the leakage currents, thus making poling ineffective. This led to decreases of the $P_{r}, d_{33}$ and $\gamma$. The exceptional increase of the $P_{r}$ from 1185 to $1195{ }^{\circ} \mathrm{C}$ was due to a large leakage current which resulted in a semiroundish shaped, lossy hysteresis loop. ${ }^{[17]}$ The data of resistivity, relative permittivity $\left(\varepsilon_{r}\right)$ and dielectric loss $(\tan \delta)$ of the $\mathrm{KNBNNO}$ (sintered at $1165{ }^{\circ} \mathrm{C}$ ) confirmed that its ferroelectric behavior was due to the intrinsic phenomena rather than mobility of the charges. Table 1 also lists the energy conversion efficiency ( $\eta_{\text {piezo }}$, only considering the material aspect without any mechanical influence) via the piezoelectric effect calculated from Equation 4, where $Q_{M}$ is the mechanical quality factor and $f_{r}$ and $f_{a}$ are resonant and anti-resonant frequencies, 


\section{WILEY-VCH}

respectively. ${ }^{[18]}$ Despite of the fact that $P_{r}$ was reduced to nearly half, the $\eta_{\text {piezo }}$ of the KNBNNO was only slightly decreased compared to that of pure KNN, showing a similar situation to that of $d_{33}$ and $\gamma$.

$\eta_{\text {piezo }}=\frac{Q \cdot k}{2+Q \cdot k} ; Q=Q_{M}+\tan ^{-1} ; k=\frac{1}{\frac{f a^{2}}{f a^{2}-f_{r}{ }^{2}}-1}$

Figure 2(a) shows the photocurrent response of the KNBNNO thick-film under a deuterium and/or tungsten-halogen fiber light probe with a spot diameter of $5 \mathrm{~mm}$. Both the deuterium and halogen lights were able individually to activate the internal charge carriers of the material and thus generate observable photocurrents. Despite the fact that the deuterium light contained a complete above-bandgap spectrum while only $40 \%$ of the halogen light was above the bandgap energy, the two light sources generated equal values of the photocurrent. As the halogen light consisted of both the visible and infrared spectrum, and considering the measured bandgap of $1.6 \mathrm{eV}$, the effective conversion of the visible light to electricity using the KNBNNO composition can be confirmed. It was observed that, when turning the deuterium light on and off, the photocurrent first changed to the opposite direction before recovering to the expected levels. A possible reason is that the photon energies carried by ultraviolet light (deuterium light) might be large enough to misalign the ferroelectric domains under an elevated energy status. In such a case, some charges trapped at the interfaces and surfaces of the domains/material might be de-trapped, inducing an instantaneously reversed current. This current would be quickly compensated by the activated photocurrent, making the overall current recover to the expected level. Vice versa, the domains returned to their original alignment when the energy status reverted to a relatively low level (ultraviolet removed), again trapping some charges and inducing another instantaneously reversed current. ${ }^{[7,19]}$

Figure 2(b) shows the photocurrent density-voltage curve ( $J$ - $V$ curve) of the KNBNNO thickfilm under a $20 \mathrm{~W}$ white-light bulb. The open-circuit voltage $\left(V_{o c}\right)$ and short-circuit current density $\left(J_{s c}\right)$ were measured to be approximately $2.4 \mathrm{~V}$ and $2 \mathrm{nA} \mathrm{cm}^{-2}$, respectively. More 


\section{WILEY-VCH}

detailed characterization of the energy conversion efficiency will be carried out with optimization of dimensions (e.g. through nano-layering) or interfaces. ${ }^{[20]}$ For a general comparison, the $V_{o c} \times J_{s c}$ value (which may indirectly reflect the output power and efficiency) of the KNBNNO sample ( $90 \mu \mathrm{m}$ thick-film) under a $20 \mathrm{~W}$ white-light bulb was $4.8 \mathrm{nA} \mathrm{V} \mathrm{cm}{ }^{-2}$, while that of a $\left(\mathrm{KNbO}_{3}\right)_{0.9}-\mathrm{BNNO}_{0.1}(20 \mu \mathrm{m}$ thick-film) under a $120 \mathrm{~W}$ tungsten-halogen lamp was only $0.07 \mathrm{nA} \mathrm{V} \mathrm{cm}{ }^{-2} \cdot{ }^{[7]}$ The difference was due to the greater ferroelectric response and better poling of the $\mathrm{KNBNNO}$ compared to those of the $\left(\mathrm{KNbO}_{3}\right)_{0.9-}-\mathrm{BNNO}_{0.1}$ in literature. ${ }^{[7]}$

Figure 2(c) shows the measurements combining the photocurrent and pyro-current extraction. Under a $20 \mathrm{~W}$ white-light bulb and with a quasi-constant temperature, an average photocurrent density of $0.3 \mathrm{nA} \mathrm{cm}^{-2}$ was obtained. In a dark environment and with a temperature fluctuation, the maximum pyro-current density of about $0.5 \mathrm{nA} \mathrm{cm}^{-2}$ was generated. Under the same white light and with the same temperature fluctuation, the maximum photo-pyro-current of about $0.8 \mathrm{nA} \mathrm{cm}^{-2}$ was achieved. In fact, the photo-pyro-current at any point was found to be exactly the sum of the individual photo- and pyro-currents if considering acceptable errors. The currents involving pyroelectric effect correspond very well to the temperature change rate. This is firm evidence that the pyroelectric and photovoltaic responses were not hindering each other. Hence, both the solar and thermal (temperature variation) energy sources could be harvested at the same time using only one material (and structure). The individual photovoltaic or pyroelectric contributions could provide an add-on value above each other, giving an immediate boost to the overall energy conversion efficiency with zero-decrement of the unit volume efficiency of each energy source.

Figure 2(d) shows an even more comprehensive characterization, in which all piezo-, pyroand photo-currents were measured simultaneously. In this measurement, the light source was provided by the same $20 \mathrm{~W}$ white-light bulb; the heat source was provided by a hairdryer; and the kinetic energy was applied by manually impacting on the surface of the sample using the tip of a pair of tweezers. When the light was turned on, the photocurrent was generated as earlier 


\section{WILEY-VCH}

presented. Subsequently, the hairdryer was turned on, inducing a pyro-current to be added to the photocurrent. While these conditions were maintained, the sample was impacted several times and in response several instant piezo-current signals were generated. The observed fluctuation and decrease of the pyro-current after the impacts were due to a fluctuation of heat caused by the hairdryer. Finally, the heat and light sources were turned off successively, with corresponding inverted currents generated. The inversion of the pyro-current was because, when the heat source was turned off, the temperature was decreasing and this resulted in a better alignment of the ferroelectric domains. In this case, more charges would be trapped on the surfaces/interfaces of the material/domains, inducing an inverted current. ${ }^{[10]}$ Although the AC piezoelectric response was not rectified, it was proved that the piezo- and pyro-currents can be independent of each other and can be extracted separately. ${ }^{[3]}$ Therefore, it can be stated that with the KNBNNO composition, solar, thermal and kinetic energies can be converted to electricity simultaneously and independently with the same material (and structure). The video in the supporting information shows a recording of the measurements. However, to define a meaningful overall efficiency of the KNBNNO for different energy conversions, further design as an optimized component should be undertaken in terms of kinetic-, thermal- and light-toelectricity energy conversion. ${ }^{[7,10]}$ Nevertheless, compared to the pure KNN in terms of the material aspect only, the KNBNNO was able to exhibit the same level of energy conversion efficiencies through the piezoelectric and pyroelectric effects. Meanwhile, the KNBNNO benefits from its additional visible-light photovoltaic sensitivity. Therefore, based on the same material, the overall unit-volume energy conversion efficiency as a multi-energy harvester for the KNBNNO is expected to be higher than that of an individual solar cell or piezoelectric/pyroelectric material due to the add-on effects of the different energy conversion principles, especially when one or two of the energy sources are not continuously available. In summary, the $\left(\mathrm{K}_{0.5} \mathrm{Na}_{0.5}\right) \mathrm{NbO}_{3}$ doped with $2 \mathrm{~mol} . \% \mathrm{Ba}\left(\mathrm{Ni}_{0.5} \mathrm{Nb}_{0.5}\right) \mathrm{O}_{3-\delta}$ has been proved to be capable of harvesting/detecting solar, thermal and kinetic energies/signals simultaneously by 


\section{WILEY-VCH}

using a single piece of material, within the same area of the material and covered by the same pair of electrodes. Realization of giant piezoelectric, pyroelectric and photovoltaic effects to be functional simultaneously in the same material has been proved feasible. This paves the way of the development of all-in-one multi-source energy harvesting and multi-functional sensing devices based on the simplest possible solution. Further compositional optimization and device fabrication are on-going.

\section{Experimental Section}

Fabrication of ceramics: Bulk ceramics of $\left(\mathrm{K}_{0.5} \mathrm{Na}_{0.5} \mathrm{NbO}_{3}\right)_{1-\mathrm{x}}-\left(\mathrm{BaNi}_{0.5} \mathrm{Nb}_{0.5} \mathrm{O}_{2.75}\right)_{\mathrm{x}}$ where $\mathrm{x}=0$, 0.02, 0.04, 0.06, 0.08 and 0.1 were fabricated via a solid-state route. Starting compositions of $\mathrm{K}_{2} \mathrm{CO}_{3}\left(\geq 99 \%\right.$, J. T. Baker), $\mathrm{Na}_{2} \mathrm{CO}_{3}$ ( $\geq 99 \%$, Sigma-Aldrich), $\mathrm{BaCO}_{3}$ (99.98 \%, Aldrich Chemistry), $\mathrm{NiO}$ (99.999 \%, Aldrich Chemistry) and $\mathrm{Nb}_{2} \mathrm{O}_{5}$ (99.9\%, Aldrich Chemistry) were dried at $220^{\circ} \mathrm{C}$, weighed and then mixed and milled in a planetary ball mill for 24 hours in ethanol and with zirconia balls ( $3 \mathrm{~mm}$ diameter). The dried mixture was calcined at $850{ }^{\circ} \mathrm{C}$ for 4 hours in air and was milled again with the same procedure. The milling process reduced the particle size to $D_{50} \approx 360 \mathrm{~nm} .8 .8 \mathrm{wt} . \%$ of binder $(3.3 \mathrm{wt} . \%$ of polyvinyl alcohol dissolved in deionized water) was subsequently added to the dried calcined powder using manual granulation in a mortar. Pellets were uniaxially pressed under $62 \mathrm{MPa}$ and sintered at 1135$1195^{\circ} \mathrm{C}$ (depending on the composition) for 2 hours on Pt foil. They were buried by sacrificial powder of the same composition in a covered alumina crucible in order to inhibit volatilization of potassium. The sintered ceramics with $\mathrm{x}=0.02-0.1$ were antique bronze color. Figure $\mathbf{S} 2$ in the supporting information shows a picture of a polished ceramic sample with $\mathrm{x}=0.02$ (the KNBNNO). The relative density of the samples was $98 \pm 0.5 \%$ (theoretical density $\approx$ $4.52 \mathrm{~g} \mathrm{~cm}^{-3}$ ). The surfaces of the ceramic samples were polished to a roughness of $50-60 \mathrm{~nm}$, followed by electrode deposition on both sides (Ag paste, DT 1402 , Heraeus, fired at $600{ }^{\circ} \mathrm{C}$ for 20 minutes). These ceramic samples were used for the ferroelectric, piezoelectric and 


\section{WILEY-VCH}

pyroelectric characterizations. Figure 3(a) and (b) show the SEM images of the fracture and the polished and thermally etched surfaces of the KNBNNO ceramic sample, respectively. The observable voids were believed to be due to:

(1) during the thermal etch at $100-150{ }^{\circ} \mathrm{C}$ for 1 hour below the sintering temperature the grain boundaries were diffused;

(2) the high etching temperature accelerated the potassium volatilization.

Figure 3(c) is a SEM image with the same magnification of the same sample after polishing but before thermal etching, where no voids are observable, consistent with the high relative density mentioned above.

Machining of thick-films: Some polished ceramic samples were machined with a laser (ProtoLaser U3, LPKF Laser \& Electronics AG, Garbsen, Germany), from which thick-films with a diameter of $5 \mathrm{~mm}$ and thickness of about $90 \mu \mathrm{m}$ were obtained. The surface after laser machining, with a roughness of $<3 \mu \mathrm{m}$, was coated with a $200 \mathrm{~nm}$ thick Au electrode. The unmachined (polished) surface was coated with a $200 \mathrm{~nm}$ thick film of indium tin oxide (ITO, transparent electrode). Both the Au and ITO films were applied by sputtering. Figure 3(d) and (e) show the SEM images of the surface after laser machining with a top view and a view from an angle of $20^{\circ}$ with the plane of the surface, respectively. Figure S3 in the supporting information shows a picture of a thick-film sample with electrodes.

Poling: Both the bulk ceramic and thick-film samples were poled in silicone oil at room temperature with a DC electric field of $90 \mathrm{kV} \mathrm{cm}^{-1}$ for 10 minutes. After poling, the two electrodes of each sample were electrically shorted for 24 hours in order to remove the trapped charges. Particularly, for thick-film samples, the laser-machined surface (with Au electrode) was connected with the positive polarity.

Characterization: X-ray diffraction (XRD, D8 Discover, Bruker, Karlsruhe, Germany) and scanning electron microscopy (SEM, Ultra Plus, ZEISS, Jena, Germany) were used for microstructural analyses. A UV-Vis-NIR spectrophotometer (Cary 500 Scan, Varian, Palo Alto, 


\section{WILEY-VCH}

USA) was used for bandgap identification. A ferroelectric test system (Precision LCII, Radiant Technologies, Inc., Albuquerque, USA) was used to characterize the ferroelectric properties. An impedance analyzer (4294A, Agilent, Santa Clara, USA) combined with a piezoelectric evaluation system (aixPES, aixACCT, Aachen, Germany) were used to characterize the dielectric and piezoelectric properties with controlled temperature. A fiber light source probe (DH-2000, Ocean Optics, Dunedin, USA) consisting of a deuterium lamp (output power $217 \mu \mathrm{W}$, wavelength $210-400 \mathrm{~nm}$ ) and a tungsten-halogen lamp (output power $295 \mu \mathrm{W}$, wavelength $300-1500 \mathrm{~nm}$ ), and a $20 \mathrm{~W}$ white-light bulb, were used to activate the photocurrent. A sample stage (LTS 350, Linkam Scientific Instruments, Tadworth, UK) and a high-precision electrometer (B2985A, Keysight, Santa Rosa, USA) were used to control the temperature and to collect data of the piezo-, pyro- and photo-currents. The temperature fluctuation was also applied through the LTS 350 stage and precisely controlled with software. When measuring the piezo-, pyro- and photo-currents, the ITO and Au electrodes of the thick-films were directly connected with the electrometer via connecting probes on the sample stage. No additional rectifier or other circuitry was integrated. The $J-V$ curves were measured with the 2450 SourceMeter (Keithley, USA) under the $20 \mathrm{~W}$ white-light bulb.

\section{Supporting Information}

Supporting Information is available from the Wiley Online Library or from the author.

\section{Acknowledgements}

The authors acknowledge Center of Microscopy and Nanotechnology of University of Oulu for the use of their facilities and the fabrication of thin film electrodes. This work has received funding from the European Union's Horizon 2020 research and innovation program under the Marie Sklodowska-Curie grant agreement number ' 705437 ', Author J. J. acknowledges the funding of the Academy of Finland (project numbers 267573, 273663 and 298409). Author P. T. acknowledges the financial support of the Ministry of Education, Youth and Sports of the Czech Republic under the project CEITEC 2020 (LQ1601).

\section{References}

[1] S. Sudevalayam, P. Kulkarni, IEEE Communications Surveys and Tutorials 2011, 13, 443. 


\section{WILEY-VCH}

[2] a) C. Xu, X. D. Wang, Z. L. Wang, J. Am. Chem. Soc. 2009, 131, 5866; b) C. Xu, Z. L. Wang, Adv. Mater. 2011, 23, 873; c) B. Yang, C. Lee, W. L. Kee, S. P. Lim, Journal of Micro-Nanolithography Mems and Moems 2010, 9, 10.

[3] Y. Yang, H. L. Zhang, G. Zhu, S. Lee, Z. H. Lin, Z. L. Wang, Acs Nano 2013, 7, 785.

[4] a) S. Lemey, F. Declercq, H. Rogier, Proceedings of the IEEE 2014, 102, 1833; b) J.

H. Lee, K. Y. Lee, M. K. Gupta, T. Y. Kim, D. Y. Lee, J. Oh, C. Ryu, W. J. Yoo, C. Y. Kang, S. J. Yoon, J. B. Yoo, S. W. Kim, Adv. Mater. 2014, 26, 765.

[5] Y. Bai, T. Siponkoski, J. Peräntie, H. Jantunen, J. Juuti, Appl. Phys. Lett. 2017, 110, 063903.

[6] R. Lopez, R. Gomez, J. Sol-Gel Sci. Techn. 2012, 61, 1.

[7] I. Grinberg, D. V. West, M. Torres, G. Y. Gou, D. M. Stein, L. Y. Wu, G. N. Chen, E. M. Gallo, A. R. Akbashev, P. K. Davies, J. E. Spanier, A. M. Rappe, Nature 2013, 503, 509.

[8] J. Rani, P. K. Patel, N. Adhlakha, H. Singh, K. L. Yadav, S. Prakash, J. Mater. Sci. Techn. 2014, 30, 459.

[9] S. Kazim, M. K. Nazeeruddin, M. Gratzel, S. Ahmad, Angew. Chem., Int. Ed. 2014, $53,2812$.

[10] C. R. Bowen, H. A. Kim, P. M. Weaver, S. Dunn, Energy \& Environmental Science 2014, 7, 25.

[11] F. G. Wang, I. Grinberg, A. M. Rappe, Appl. Phys. Lett. 2014, 104, 4.

[12] J. F. Scott, M. Dawber, Appl. Phys. Lett. 2000, 76, 3801.

[13] a) S. J. Zhang, J. B. Lim, H. J. Lee, T. R. Shrout, IEEE T. Ultrason. Ferr. 2009, 56, 1523; b) R. G. Sabat, B. K. Mukherjee, W. Ren, G. M. Yang, J. Appl. Phys. 2007, 101, 7.

[14] H. Birol, D. Damjanovic, N. Setter, J. Eur. Ceram. Soc. 2006, $26,861$.

[15] H. Birol, D. Damjanovic, N. Setter, J. Am. Ceram. Soc. 2005, 88, 1754.

[16] U. Fluckiger, H. Arend, H. R. Oswald, Am. Ceram. Soc. Bull. 1977, 56, 575.

[17] E. Mostafavi, A. Ataie, M. Ahmadzadeh, M. Palizdar, T. P. Comyn, A. J. Bell, Mater. Chem. Phys. 2015, 162, 106.

[18] C. D. Richards, M. J. Anderson, D. F. Bahr, R. F. Richards, J. Micromech. Microeng. 2004, 14, 717.

[19] a) S. Liu, F. Zheng, I. Grinberg, A. M. Rappe, J. Phys. Chem. Lett. 2016, 7, 1460; b) Q. Shen, Y. Cao, S. Liu, L. Gan, J. M. Li, Z. X. Wang, J. S. Hui, X. F. Guo, D. S. Xu, Z. F. Liu, J. Phys. Chem. Lett. 2010, 1, 1269; c) J. Park, S. S. Won, C. W. Ahn, I. W. Kim, J. Am. Ceram. Soc. 2013, 96, 146.

[20] a) F. G. Wang, S. M. Young, F. Zheng, I. Grinberg, A. M. Rappe, Nat. Commun. 2016, 7, 7; b) J. H. Heo, S. H. Im, Adv. Mater. 2016, 28, 5121.

Received: ((will be filled in by the editorial staff))

Revised: ((will be filled in by the editorial staff)) Published online: ((will be filled in by the editorial staff)) 
(a)

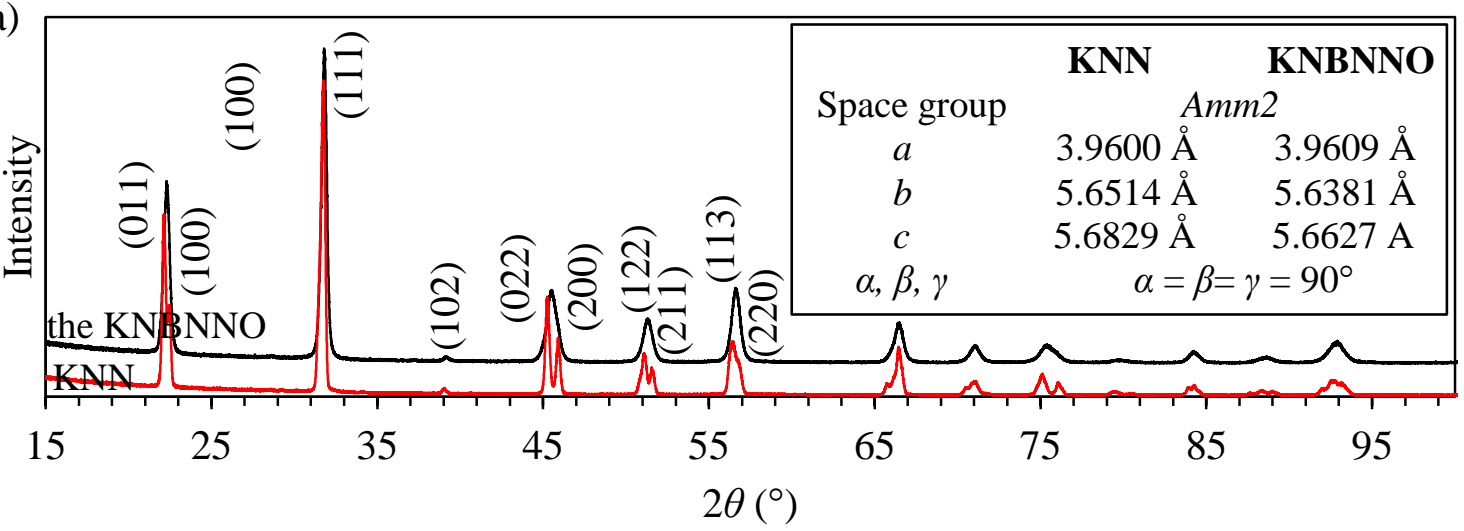

(b)

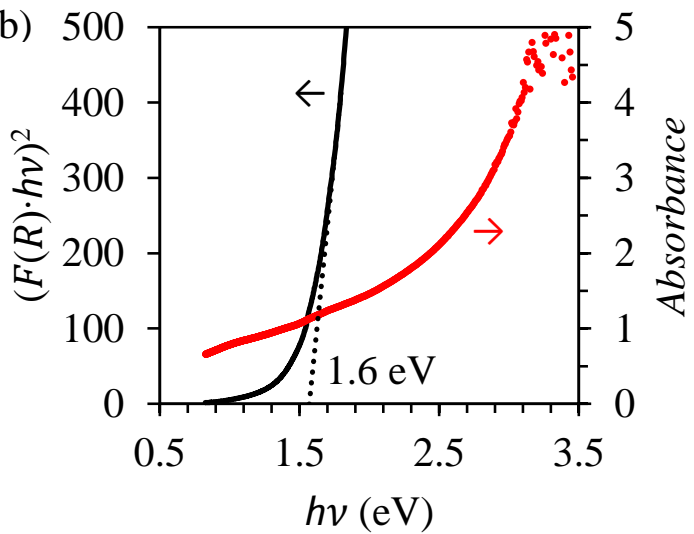

(d)

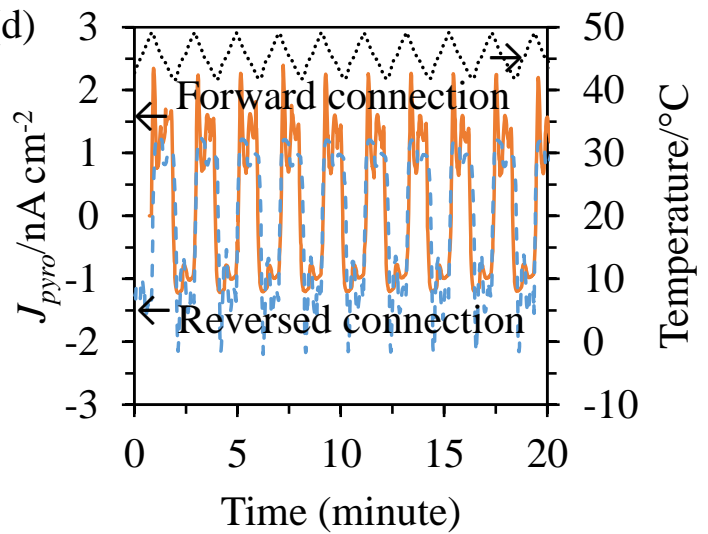

(c)

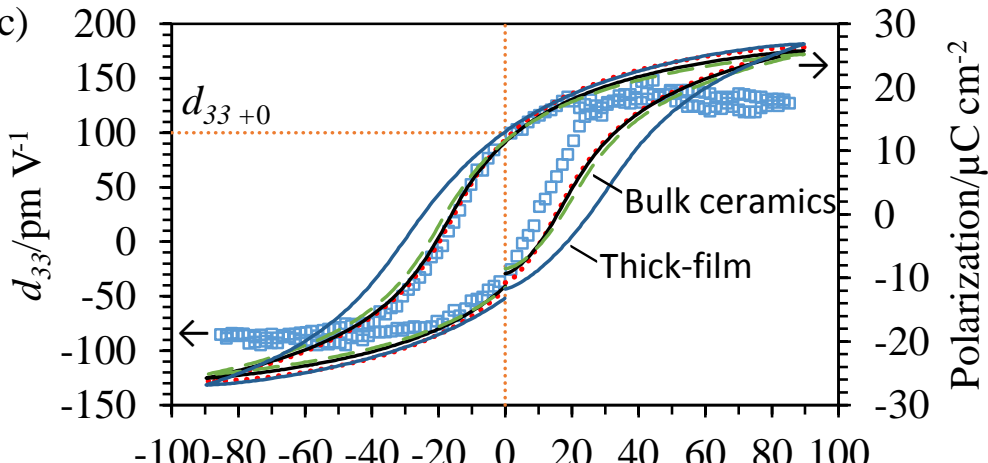

Electric field $\left(\mathrm{kV} \mathrm{cm}^{-1}\right)$

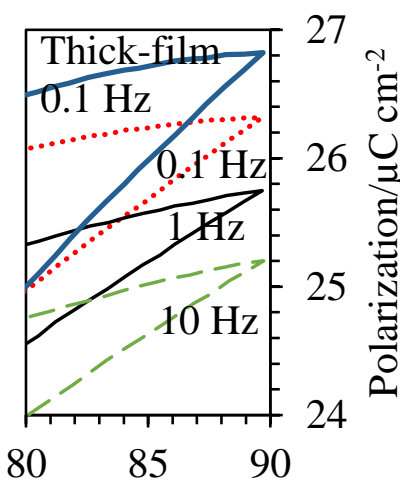

Electric field $\left(\mathrm{kV} \mathrm{cm}^{-1}\right)$

Figure 1. (a) XRD pattern of the sintered ceramics; (b) Optical absorption and reflection spectra and band gap determination of the thick-film; (c) Dependence of polarization (FE loops, measured with different frequencies) and converse piezoelectric coefficient on electric field for the ceramics and thick-films; and (d) Response of pyroelectric current of the ceramics generated with temperature fluctuation. 

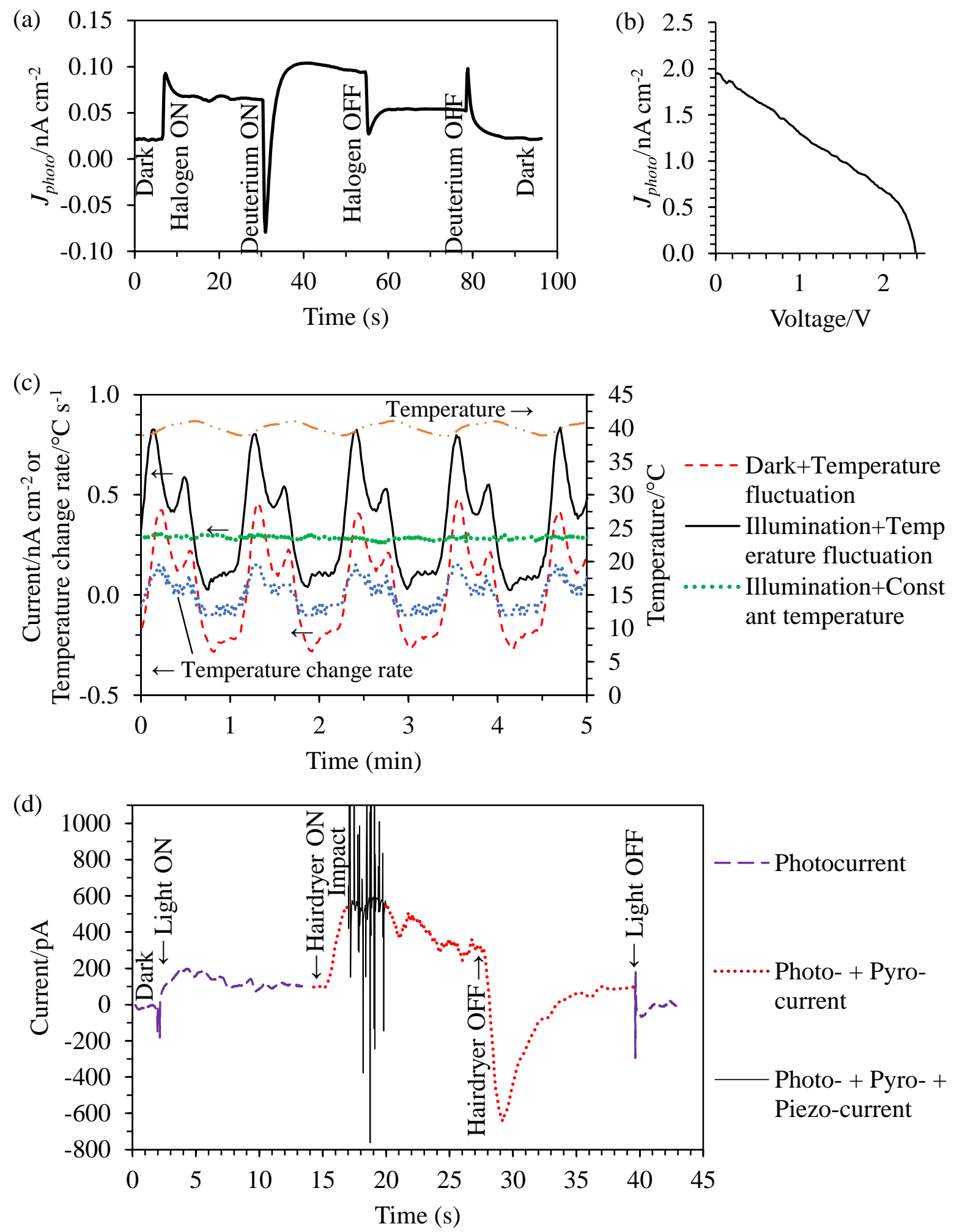

Figure 2. (a) The photocurrent of the thick-film generated under the deuterium and tungstenhalogen fiber light source; (b) The $J$ - $V$ curve of the thick-film measured under the $20 \mathrm{~W}$ white light; (c) The current (photo- and/or pyro-) of the thick-film generated with different environmental conditions; and (d) The photo-, pyro- and piezo-currents of the thick-film generated individually and/or simultaneously from light, thermal and kinetic energy sources. 


\section{WILEY-VCH}

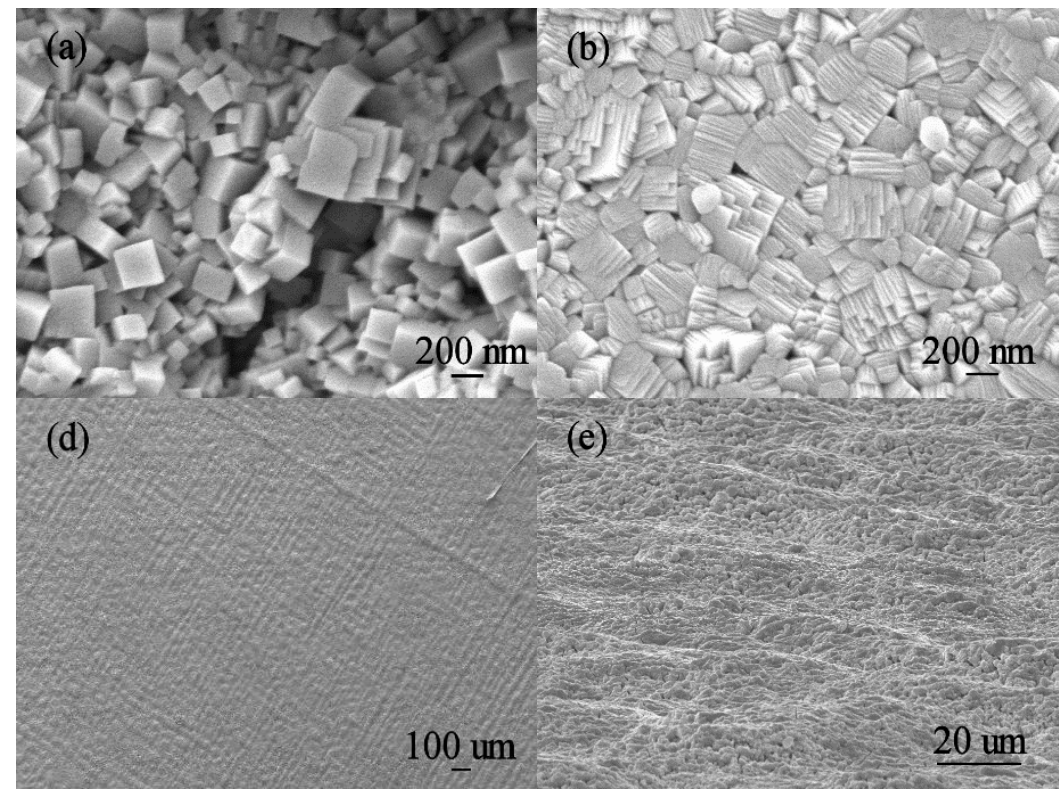

(c)

$200 \mathrm{~nm}$

Figure 3. The SEM images of the (a) fracture surface, (b) polished and thermally etched surface and (c) polished but not etched surface of the ceramics, and the surface after laser machining of the thick-films with (d) a top view and (e) a view from an angle of $20^{\circ}$ with the plane of the surface.

Table 1. Summary of the material and functional properties of KNN doped with different amount of BNNO and sintered at different temperatures.

\begin{tabular}{|c|c|c|c|c|c|c|c|c|c|c|}
\hline $\begin{array}{c}\text { Concentra- } \\
\text { tion of } \\
\text { BNNO } \\
\text { [mol. \%] }\end{array}$ & $\begin{array}{c}\text { Sintering } \\
\text { temperat- } \\
\text { ure }\left[{ }^{\circ} \mathrm{C}\right]\end{array}$ & Phase $^{\text {a) }}$ & $\begin{array}{c}\text { Bandgap } \\
{[\mathrm{eV}]}\end{array}$ & $\begin{array}{c}P_{r}(1 \mathrm{~Hz}) \\
{\left[\mu \mathrm{C} \mathrm{cm}{ }^{-2}\right]}\end{array}$ & $\begin{array}{c}\text { Resisti- } \\
\text { vity } \\
{[\mathrm{G} \Omega} \\
\mathrm{cm}]\end{array}$ & $\begin{array}{c}d_{33} \\
{[\mathrm{pm} / \mathrm{V}]}\end{array}$ & $\begin{array}{c}\gamma \\
{\left[\mu \mathrm{C} \mathrm{m}^{-2}\right.} \\
\left.\mathrm{K}^{-1}\right]\end{array}$ & $\begin{array}{c}\varepsilon_{r} \\
(1 \mathrm{kHz})\end{array}$ & $\begin{array}{c}\tan \delta \\
(1 \mathrm{kHz})\end{array}$ & $\eta_{\text {piezo }}$ \\
\hline 0 & 1135 & $\mathrm{O}$ & $>4$ & $20^{[7]}$ & 9.9 & $100^{[7]}$ & $140^{[7]}$ & $472^{[7]}$ & $0.04^{[7]}$ & 0.49 \\
\hline \multirow{4}{*}{2} & 1165 & 0 & 1.59 & 11.3 & 52 & 100 & 128 & 934 & 0.025 & 0.44 \\
\hline & 1175 & $\mathrm{O}$ & 1.63 & 6.5 & 19 & 66 & 77 & 863 & 0.028 & 0.44 \\
\hline & 1185 & $\mathrm{O}$ & 1.58 & 5.6 & 5.4 & 57 & 31 & 812 & 0.029 & 0.43 \\
\hline & 1195 & $\mathrm{O}$ & 1.58 & 7.2 & 2.4 & 41 & 15 & 796 & 0.044 & - \\
\hline \multirow{3}{*}{4} & 1175 & $\mathrm{O}$ & 1.55 & 0.9 & 55 & $<10$ & 9.3 & 660 & 0.022 & - \\
\hline & 1185 & $\mathrm{O}$ & 1.50 & 0.9 & 55 & $<10$ & 6.2 & 659 & 0.030 & - \\
\hline & 1195 & $\mathrm{O}$ & 1.50 & 4.3 & 3.4 & $<10$ & 6.0 & 684 & 0.040 & - \\
\hline \multirow{2}{*}{6} & 1185 & $\mathrm{~T}$ & 1.58 & 0.6 & 102 & -b) & 3.1 & 639 & 0.017 & - \\
\hline & 1195 & $\mathrm{~T}$ & 1.56 & 0.6 & 58 & - & 3.9 & 636 & 0.025 & - \\
\hline 8 & 1195 & $\mathrm{~T}$ & 1.56 & 0.6 & 68 & - & 3.1 & 668 & 0.022 & - \\
\hline 10 & 1195 & C & 1.16 & $\approx 0$ & 133 & - & n.a. & 632 & 0.016 & - \\
\hline
\end{tabular}

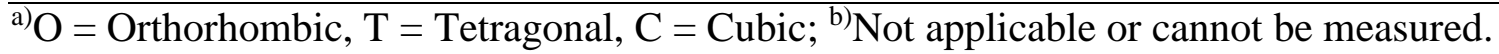




\section{WILEY-VCH}

A multi-functional perovskite composition exhibiting giant ferroelectricity and narrow bandgap is reported. Ceramics and thick-films are fabricated via solid-state reaction and laser machining, respectively. Their balanced piezoelectric, pyroelectric and photovoltaic properties and the multi-task feature pave the way to the development of all-in-one multi-source energy harvesting and multi-functional sensing devices based on the simplest solution of materials and structures.

Hybrid energy harvesting, piezoelectric, pyroelectric, photovoltaic, multi-functional

Yang Bai*, Pavel Tofel, Jaakko Palosaari, Heli Jantunen and Jari Juuti

Game Changer: A Multi-functional Perovskite Exhibiting Giant Ferroelectricity and Narrow Bandgap with Potential Application in a Truly Monolithic Multi-energy

Harvester or Sensor

ToC figure

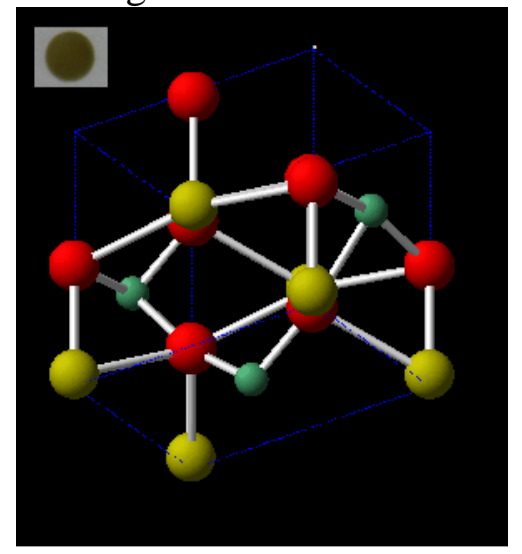




\section{WILEY-VCH}

Copyright WILEY-VCH Verlag GmbH \& Co. KGaA, 69469 Weinheim, Germany, 2016.

\section{Supporting Information}

Game Changer: A Multi-functional Perovskite Exhibiting Giant Ferroelectricity and Narrow Bandgap with Potential Application in a Truly Monolithic Multi-energy Harvester or Sensor

Yang Bai*, Pavel Tofel, Jaakko Palosaari, Heli Jantunen and Jari Juuti

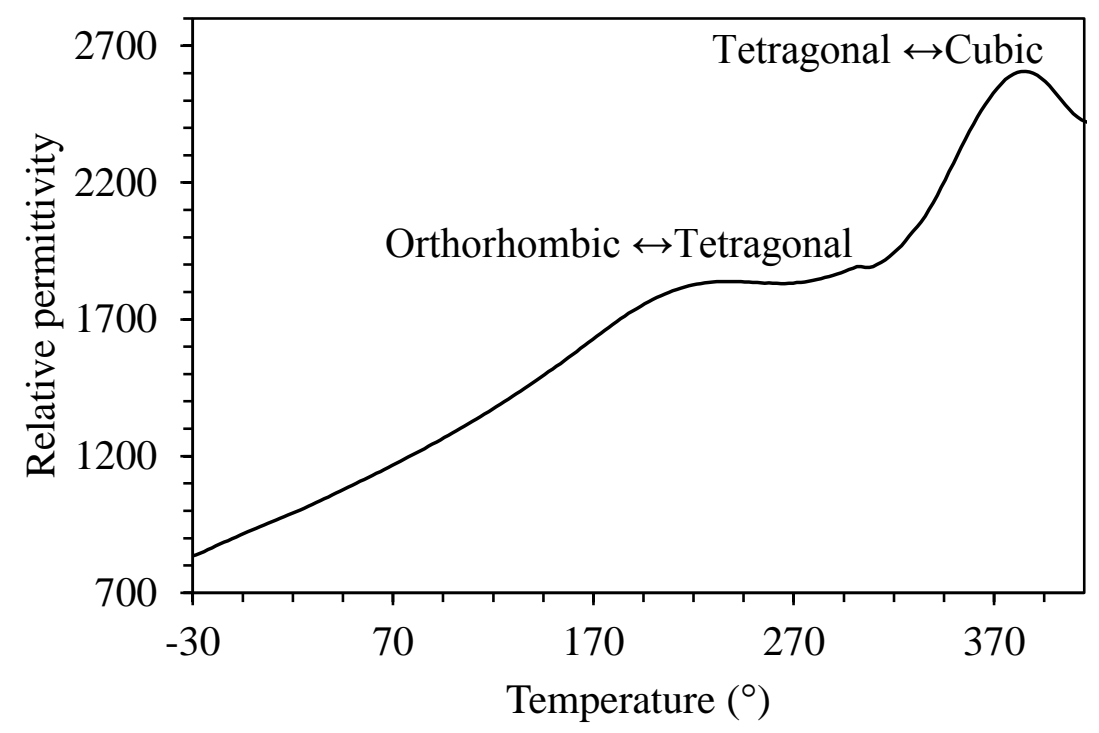

Figure S1. Dependence of relative permittivity on temperature for the ceramics. The two fused peaks imply the orthorhombic to tetragonal and tetragonal to cubic phase transitions.

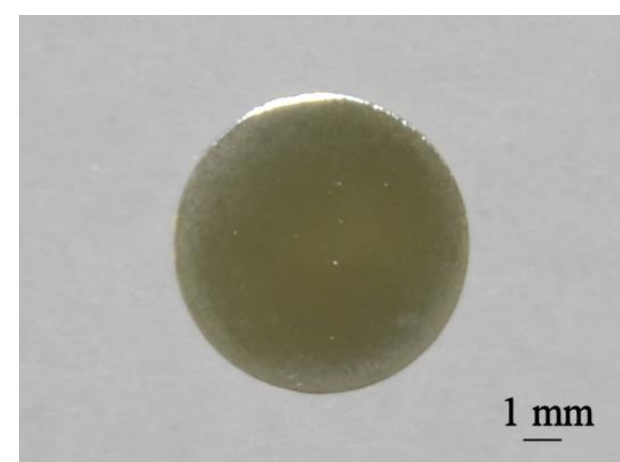

Figure S2. Picture of a polished ceramic sample. 


\section{WILEY-VCH}

(a)

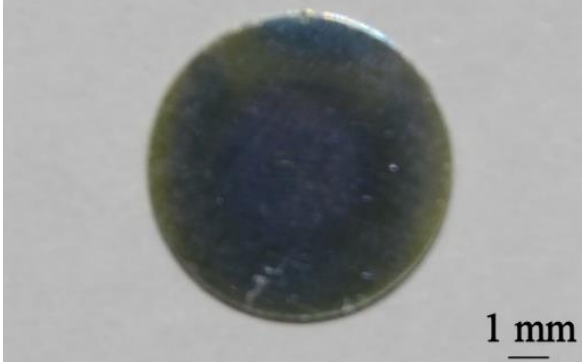

(b)

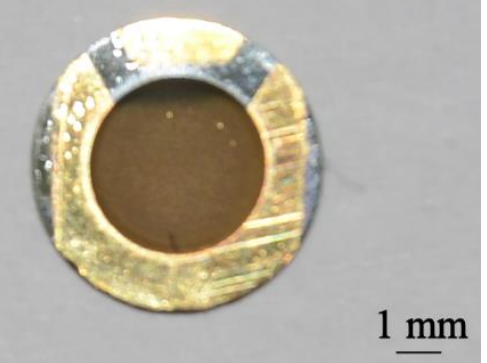

Figure S3. Pictures of a thick-film sample: (a) the polished surface with ITO electrode deposition and (b) the surface after laser machining covered with Au electrode.

Table S1. Parameters used in the ferroelectric and converse effect piezoelectric measurements

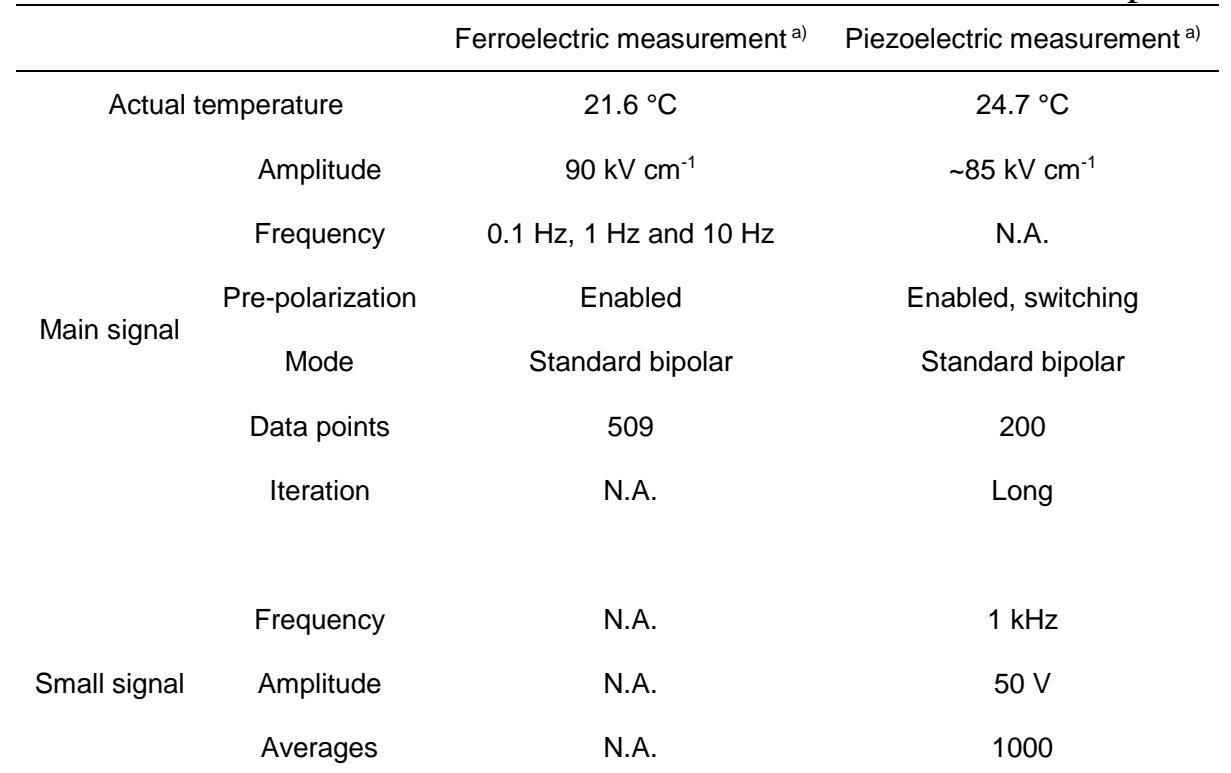

${ }^{\mathrm{a}}$ The equipment used for the measurements are mentioned in the experimental section. 